\title{
Imagination entachée de singularité versus conception immaculée : De l'irréductibilité des objets intentionnels à de simples concepts
}

\author{
Par BRUNO LECLERCQ \\ Université de Liège
}

Résumé En repartant des analyses de Jacques English sur la triple modalité (perceptive, imaginative et signitive) de l'intentionnalité, je m'attacherai à opposer, parmi les deux modalités qui s'abstraient du réel pour envisager des possibles, l'imagination, qui les traite sur le mode de la donation singulière et, relevant de l'intuition, fournit dès lors de véritables objets intentionnels susceptibles de jugements synthétiques, et la conception, qui les traite sur le mode de la visée signitive générale et, pour cette raison, ne fournit que des concepts, qui ne sont susceptibles que de jugements analytiques et ne sont pas encore d'authentiques objets intentionnels.

\section{Introduction}

Quoi qu'en dise Jacques Derrida ${ }^{1}$, la phénoménologie husserlienne n'est pas essentiellement une pensée de la pleine présence. En dépit de l'importance des motifs du "retour aux choses mêmes », de la donation de la chose en personne ( en chair et en os »), ou encore du remplissement intuitif (non seulement perceptif, mais aussi catégorial ou eidétique), la phénoménologie est aussi et surtout une pensée de l'inscription systématique des données sensorielles directement vécues dans un horizon de contenus simultanés, passés et futurs qui ne sont pas eux-mêmes immédiatement présents à la conscience; et dès lors pensée de l'intentionnalité entendue comme rapport

${ }^{1}$ J. Derrida, La voix et le phénomène, Paris, P.U.F., 1967, p. 3, 14-16, 43-48, 56-77, 111-117. 
de la conscience à des objets qui ne lui sont pas immanents ; enfin de l'interaction entre donation effective et simple visée "à vide» de ces objets. Comme les travaux d'autres disciples de Brentano, en particulier Meinong, la phénoménologie husserlienne est profondément marquée par l'idée que la représentation ne se réduit pas à la perception des réalités sensibles - et moins encore à la simple présence de contenus sensoriels à la conscience qu'il y a précisément d'autres types d'objets que les seules réalités, et en outre d'autres rapports intentionnels aux objets que la seule perception, et qu'il semble même y avoir des représentations " sans objet» (quoique pas sans contenu). En outre, la phénoménologie est, très explicitement, une étude, non des vécus effectifs de telle ou telle conscience, mais des lois régissant les phénomènes, ce qui suppose de ne pas seulement s'intéresser à ce qui est effectivement vécu, mais à ce qui peut être vécu, aux vécus possibles. Comme c'était le cas de la psychologie descriptive brentanienne, qui reposait sur une "perception interne » irréductible aux impressions de réflexion lockiennes, cette eidétique de la conscience suppose donc bien autre chose qu'un simple retour réflexif de la conscience sur les vécus qu'elle vient tout juste d'éprouver. Préoccupée de l'essence (et des lois d'essence) des vécus, la phénoménologie suppose une visée idéalisante, dite " réduction eidétique ». En régime transcendantal, la réduction eidétique s'appuiera même explicitement sur une épochè, qui suspend tout simplement la question de la réalité effective des phénomènes étudiés ${ }^{1}$; ces derniers seront clairement étudiés pour leur valeur phénoménologique et non pour leur effectivité. Or, tout cela indique qu'à côté de la perception et du remplissement intuitif qu'elle permet, d'autres modalités de la conscience sont absolument centrales au projet même de la phénoménologie.

Suivant sur ce point Victor Delbos, Jacques English a notoirement soutenu qu'il y a, chez Husserl, essentiellement trois modalités de la conscience représentative (par opposition aux modes intentionnels qui s'appuient sur la représentation, tels que le jugement, le désir ou la crainte), à savoir la

${ }^{1}$ E. Husserl, Ideen zu einer reinen Phänomenologie und phänomenologischen Philosophie, 1950, §§ 33-34, 50, 75 [Hua III, p. 69-76, 118-119, 171-174], trad. fr. Idées directrices pour une phénoménologie I, Paris, Gallimard, 1950, p. 105-111, 164-167, 238-241. Soulignons d'emblée que la modification-de-phantasia ne s'identifie pas à cette modification-de-neutralité (voir sur ce point l'introduction de l'éditeur au vol. XXIII des Husserliana [Hua XXIII, p. LXVI-LXX], trad. fr. Edmund Husserl, Phantasia, conscience d'image, souvenir, Grenoble, Millon, 2002, p. 35$38)$. 
perception, l'imagination et la signification ${ }^{1}$. Or, à cet égard, les deux dernières modalités ont précisément pour spécificité de prétendre s'abstraire de ce qui est effectivement donné pour pouvoir représenter ce qui est seulement possible. Je peux m'imaginer portant ici même, devant vous, un chapeau haut-de-forme bien qu'il n'en soit effectivement rien, et je peux concevoir l'idée générale - viser la signification — « licorne » même s'il n'y a effectivement aucun individu réel conforme à cette idée. Cependant, si l'imagination et la simple intention de signification se distinguent ainsi toutes deux nettement de la perception, laquelle est contrainte par ce qui est effectivement donné, c'est selon nous dans l'opposition qu'il y a cette fois entre imagination et simple intention de signification que se jouent une bonne part des enjeux du projet phénoménologique. Du point de vue de la psychologie descriptive, ce n'est pas du tout phénoménologiquement la même chose que

\footnotetext{
${ }^{1}$ J. English, Sur l'intentionnalité et ses modes, Paris, P.U.F, 2006, p. 52-53. Pour English, la phénoménologie se caractérise précisément par sa thématisation de la diversité des modalités de conscience et donc des modes de relation à l'objet. Ayant constaté dans la Philosophie de l'arithmétique un saut majeur entre les représentations sensibles (fondements primaires, ...) répondant à des lois synthétiques matérielles et les objets catégoriaux susceptibles de se soumettre aux lois de l'analytique formelle, Husserl se serait mis à la recherche d'intermédiaires permettant ce passage et en serait ainsi venu à un traitement systématique des différents modes d'intentionnalité. C'est ce qu'English appelle l'étude des rapports « horizontaux » entre types d'actes (et corrélativement entre types d'objets) par opposition aux rapports « verticaux » qui lient les actes à leurs objets. Dans l'itinéraire de Husserl, cela impliqua un va-et-vient entre solutions privilégiant la modalité perceptive et solutions privilégiant la modalité signitive, deux modalités entre lesquelles la modalité imaginaire devra jouer un rôle essentiel d'intermédiaire. Dès la Philosophie de l'arithmétique, ce sont d'ailleurs des « représentations imaginaires » qui permettent de passer des représentations sensibles de pluralité à la signification logique d'ensemble. La critique de Twardowski, qui privilégiait le modèle pictural de la représentation, devait toutefois amener Husserl à rejeter provisoirement l'importance de l'imagination au profit de la seule signification, avant d'y revenir dans l'appendice à la $5^{\mathrm{e}}$ Recherche Logique et dans la $6^{\mathrm{e}}$ Recherche, puis de consacrer l'importance égale des trois modalités par ses cours sur l'imagination en 1904, sur la perception dans le temps et l'espace en 1905 et 1907 et sur la signification en 1908. English explique que, dans un contexte phénoménologique français où la modalité signitive était négligée au profit de la perception et de l'imagination, c'est la modalité signitive que lui-même a surtout dû remettre à l'honneur. Héritier pour notre part des lectures frégéennes de la phénoménologie (mais aussi des lectures brentaniennes focalisées sur la question des représentations sans objet), c'est au contraire la modalité imaginative que nous avons dû nous réapproprier.
} 
de viser l'intention de signification " montagne d'or » et d'en imaginer une. Et, du point de vue de la théorie de la connaissance, ce n'est pas du tout la même exploration des possibles que permettent ces deux modalités de la conscience, donc pas non plus les mêmes nécessités qu'elles révèlent.

\section{Concepts et objets imaginaires}

Il est en effet extrêmement significatif que Husserl range l'imagination aux côtés de la perception parmi les actes intuitifs susceptibles de remplir les intentions de signification ${ }^{1}$. Une montagne imaginée remplit l'intention de signification « montagne » même si elle ne le fait pas exactement de la même manière qu'une montagne réellement perçue. Ce qui distingue la montagne imaginée de la simple signification «montagne », c'est la richesse des éléments sensoriels - Husserl dira bientôt "hylétiques» - qui dote la première d'un contenu nettement plus dense que le seul trait conceptuel qui compose et épuise la signification «montagne ». La montagne que j'imagine a une certaine forme, certaines couleurs, et de la neige ou non sur son sommet. Tout cela lui confère une certaine singularité que n'a pas la signification (générale) «montagne ». Si l'imagination peut, comme la perception, remplir l'intention de signification, c'est parce qu'elle est une représentation singulière et qu'une représentation singulière est " pleine » de déterminations que ne comporte pas la signification générale. Sans doute, contrairement à une montagne réelle, la montagne que j'imagine reste-t-elle indéterminée en bien des points. Faute de l'avoir imaginée dans tous ses détails, je ne puis préciser le nombre de bouquetins qui parcourent ses flancs, alors que toute montagne réelle est, à chaque instant, parcourue par un nombre déterminé de bouquetins. Mais, d'un point de vue phénoménologique, la perception d'une mon-

\footnotetext{
${ }^{1}$ E. Husserl, Logische Untersuchungen, VI, §§ 6, 14b, 21-23, 45 [Hua XIX/2, p. 560, 591, 608-613, 672], trad. fr. Recherches Logiques, Paris, P.U.F., 1963, p. 40, 77, 99106, 176-177. Comme la perception, la phantasia est présentification, laquelle est toutefois libre et discontinue tandis que la première est passive et continue ([Hua XXIII, p. 561-562], trad. fr. Phantasia, conscience d'image, souvenir, op. cit., p. 526-527). Contrairement à ce que pensait Brentano, la phantasia est donc un représenter « propre» (introduction de l'éditeur, trad. fr. p. 18-20, 30 ; cf. aussi toute la réflexion sur la rétention comme modification de l'appréhension par opposition au souvenir comme reproduction p. 31-34). Quoique sur le mode du «quasi/comme si », la phantasia est, comme la perception, une intuition individuelle et remplissante (ibid., p. 38-39). Et c'est pourquoi elle aussi est contrainte par le remplissement possible ([Hua XXIII, p. 558, 563-564], trad. fr. op. cit., p. 524, 528-529).
} 
tagne réelle ne me fournit pas non plus d'emblée l'ensemble des informations que pourrait me fournir une exploration plus détaillée. C'est une thèse bien connue de la phénoménologie de la perception, que celle-ci ne donne toujours son objet que par esquisses, de sorte que la montagne perçue n'est que partiellement déterminée même si la montagne réelle l'est entièrement.

Tout ceci, qui relève de la psychologie descriptive, importe grandement lorsqu'il s'agit d'étudier le statut des corrélats intentionnels — des « contenus »- de ces différents actes que sont la perception, l'imagination ou l'intention de signification. D'importants débats ont en effet émaillé la question de savoir si les représentations de ce qui n'existe pas effectivement doivent être dites (avec contenu mais) "sans objet», comme le pense Bolzano $^{1}$, ou si, en plus d'un contenu, elles sont bien également dotées d'un objet quoique d'un objet inexistant, comme le prétendent Twardowski ${ }^{2}$ et Meinong ${ }^{3}$. Les débats se prolongent lorsqu'il s'agit de s'intéresser aux propriétés de ce qui est ainsi imaginé ou simplement conçu sans être effectivement donné dans la perception sensible - et parfois sans jamais pouvoir y être donné, comme c'est le cas du célèbre carré rond.

Pour nous être longuement intéressé ${ }^{4}$ aux logiques dites «meinongiennes », qui s'efforcent de rendre compte des conditions de vérité des jugements sur les objets inexistants (Sherlock Holmes, l'actuel roi de France, la montagne d'or, ...) ou impossibles (le carré rond), il nous faut souligner combien est insatisfaisant le traitement logique qui y est réservé à ces

\footnotetext{
${ }^{1}$ B. Bolzano, Wissenschaftslehre, Leipzig, Meiner, 1929, §§ 66-67, vol. I, p. 296305; trad. fr. partielle Théorie de la science, Paris, Gallimard, 2011, p. 191-197.

2 K.Twardowski, Zur Lehre vom Inhalt und Gegenstand der Vorstellungen, 1894, $\S 5,23-29$, trad. fr. Sur la théorie du contenu et de l'objet des représentations, dans E. Husserl - K. Twardowski, Sur les objets intentionnels (1893-1901), Paris, Vrin, 1993, p. 105-114.

${ }^{3}$ A. Meinong, Über Gegenstandstheorie, Leipzig, Barth, 1904, §§ 2-4, trad. fr. La théorie de l'objet, Paris, Vrin, 1999, p. 68-76.

${ }^{4}$ Voir en particulier B. Leclercq, «À l'impossible, nul objet n'est tenu. Statut des 'objets' inexistants et inconsistants et critique frégéo-russellienne des logiques meinongiennes », dans Sébastien Richard éd., Analyse et ontologie. Le renouveau de la métaphysique dans la tradition analytique, Paris, Vrin, 2011, p. 159-198; "Quand c'est l'intension qui compte. Opacité référentielle et objectivité », dans Bulletin d'Analyse Phénoménologique, 2010 (vol. 6, n 8), p. 83-108; «En matière d'ontologie, l'important n'est pas de gagner, mais de participer », dans Igitur, 2012 (vol. 4, n²), p. 1-24; «Faire cohabiter les objets sans domicile fixe (homeless objects). Chisholm et les logiques meinongiennes », dans Bulletin d'Analyse Phénoménologique, 2014 (vol. 10, $\mathrm{n}^{\circ}$ 6), D’un point de vue intentionnel : Aspects et enjeux de la philosophie de Roderick Chisholm (Actes n6), p. 85-111.
}

\section{4}


prétendus « objets », traitement logique qui n'est que le revers d'une analyse descriptive insuffisante en ce qui concerne ces «objets » et les actes intentionnels dont ils sont les « produits ». L'imagination et la simple intention de signification sont en effet deux modalités intentionnelles nettement distinctes, de sorte que leurs corrélats - par exemple la montagne que j'imagine et la signification " montagne » - ne sont pas du tout susceptibles des mêmes propriétés ni du même traitement logique. N'en déplaise aux meinongiens, Sherlock Holmes, l'actuel roi de France, la montagne d'or et le carré rond ne sont pas tous de la même manière des objets inexistants. Et ils ne sont pas tous de la même manière des objets incomplets - c'est-à-dire indéterminés pour certains prédicats, et violant donc à leur égard le principe de tiers exclu —, le dernier étant en outre inconsistant — c'est-à-dire déterminé par des prédicats logiquement incompatibles, et violant donc sur ce point le principe de non-contradiction. Cette manière de considérer les choses est insatisfaisante car Sherlock Holmes n'est pas du tout incomplet de la même manière que l'est la montagne d'or « en général ». En outre, nous y reviendrons, le triangle dont la somme des angles est supérieure à $180^{\circ}$ n'est pas du tout inconsistant de la même manière que l'est le triangle qui a plus de 3 côtés.

Pour les meinongiens ${ }^{1}$, en effet, c'est une spécificité des objets réels que d'être complets, c'est-à-dire déterminés pour toute paire de prédicats (pertinents) - en objet réel, j'ai ou non un nævus sur l'épaule droite tandis que les objets inexistants sont généralement incomplets - faute que Conan Doyle se soit prononcé sur cette question, Sherlock Holmes est indéterminé quant au fait qu'il a ou non un nævus sur l'épaule droite. Pionnier des logiques meinongiennes, Richard Routley rapproche explicitement ce cas de celui du triangle en général, qui est indéterminé quant à la question de savoir s'il est équilatéral, isocèle ou scalène, alors que chaque triangle effectivement existant, chaque objet réel de forme triangulaire, est forcément déterminé à l'égard de ces prédicats ${ }^{2}$. Ce rapprochement est à lui seul symptomatique de l'incapacité des logiques meinongiennes à bien distinguer le cas des objets imaginaires — corrélats

\footnotetext{
${ }^{1}$ Développée notamment par Meinong dans Über Möglichkeit und Warscheinlichkeit (Leipzig, Barth, 1915), la thèse de l'incomplétude des objets inactuels se retrouve, d'une manière ou d'une autre, chez tous les héritiers de Meinong dans les domaines de la logique et de l'ontologie.

2 R. Routley, Exploring Meinong's Jungle and Beyond, Canberra, Department Monograph \#3 of the Philosophy Department of the Australian National University, 1980, p. 92-93.
} 
d'actes d'imagination - de celui de simples concepts - corrélats d'intentions de signification. Il est dans la nature d'un concept comme celui de «triangle» d'être indéterminé sur tout trait qui n'entre pas dans sa définition ou n'est pas une conséquence analytique de celle-ci. Le triangle en général n'est ni vert ni rouge, ni grand ni petit, ni équilatéral ni scalène, parce que l'intention de signification constitutive de ce concept reste entièrement indéterminée à l'égard de toutes ces questions et c'est précisément ce qui fait sa généralité. Sherlock Holmes, par contre, est, dans l'intention de Conan Doyle comme dans celle de ses lecteurs, un objet singulier, objet que nous ne connaissons certes qu'à travers la description (conceptuelle) qu'en donnent les romans de Conan Doyle, mais qui n'entend clairement pas se réduire à cette description; Holmes, supposons-nous sans cesse, a tout un tas de propriétés que Doyle ne nous décrit pas : entre les moments que décrit Doyle, Holmes est bien quelque part, même si nous ne savons pas exactement où, et sa célèbre casquette de voyage à rabats doit bien avoir une forme et une couleur très précise, même si Doyle ne nous l'indique pas précisément...

En fait, nous supposons sans cesse que, comme les êtres humains réels, Holmes est un objet complet. À cet égard, l'analyse de logiciens meinongiens comme Terence Parsons, qui fait des personnages de fiction des objets incomplets possédant toutes et rien que les propriétés que leur attribue l'œuvre littéraire qui les met en scène ${ }^{1}$, passe clairement à côté du caractère singulier de ces personnages. Ceux-ci ne sont pas de simples significations générales caractérisées par l'ensemble des traits définitoires que précise l'ouvre littéraire, laquelle serait alors tout entière définition du concept « Sherlock Holmes »; ils sont des objets singuliers partiellement décrits par l'œuvre. Certes, l'œuvre n'énonce-t-elle pas les propriétés de réalités qui lui préexisteraient et seraient ontologiquement indépendantes d'elle, mais elle se présente néanmoins comme la description d'entités singulières imaginaires qui transcendent ces descriptions et possèdent nettement plus de propriétés que celles qui sont explicitement énoncées.

À cet égard, la différence entre le triangle en général et Sherlock Holmes est majeure. Le premier est une signification générale caractérisée par des traits définitoires - figure fermée à trois côtés — et il n'a, nous devrons y revenir, d'autre "propriété » que celles qui résultent nécessairement de ces traits définitoires. Le second est un objet singulier, qui trouve certes son origine dans une description conceptuelle, mais ne prétend pas s'y réduire ; on suppose au contraire qu'il possède tout un tas de propriétés sur

${ }^{1}$ T. Parsons, Nonexistent objects, New Haven \& London, Yale University Press, 1980, p. 55-56. 
lesquelles Doyle reste silencieux et qu'on ne peut pas simplement déduire de ce qu'il dit de Holmes. Le triangle en général et Sherlock Holmes ne sont pas incomplets de la même façon. Le premier se limite par nature à un certain nombre de traits définitoires et à l'ensemble de leurs conséquences nécessaires. Le second ne se réduit pas à une définition mais possède un ensemble vaste de propriétés de toutes sortes, sur lesquelles toutefois Doyle ne nous a communiqué qu'une information limitée. Comme dans le cas de l'objet réel partiellement perçu, l'incomplétude est ici épistémique; nous ne connaissons pas certaines des propriétés de Holmes. Si, comme le suggère Doyle, Holmes est un être humain singulier, et non la signification générale "détective habitant au 221b Baker Street», il doit être vrai ou faux de lui qu'il a un nævus sur l'épaule droite même si nous ignorons ce qu'il en est.

Loin que son incomplétude le rapproche des notions générales, l'objet imaginaire Holmes est, sur ce point, plutôt similaire aux êtres humains réels ; de la plupart de ceux-ci, nous ne savons pas s'ils ont ou non un nævus à l'épaule droite, mais cette incomplétude de notre savoir à leur égard n'en fait pas pour autant des objets qui sont eux-mêmes incomplets; il ne faut pas confondre incomplétude épistémique et incomplétude ontologique. Une différence, bien sûr, subsiste dans le fait que nous pouvons, dans le cas des objets réels, poursuivre l'investigation empirique et réduire l'incomplétude épistémique, tandis que nous ne pouvons effectuer le même travail pour un être fictif comme Sherlock Holmes. Dans son cas, l'incomplétude épistémique ne peut être réduite qu'au moyen... de l'imagination; soit qu'il s'agisse de l'imagination de Doyle, qui peut déterminer toujours davantage le personnage et exprimer ces nouvelles déterminations dans d'autres descriptions, soit qu'il s'agisse de l'imagination des lecteurs qui peuvent à leur tour déterminer le personnage par des images singulières qui précisent les descriptions de Doyle là où elles restent encore générales... Bien que, par sa liberté et sa créativité, l'imagination se distingue évidemment assez nettement de la perception sensible, qui est pour sa part passive et contrainte par le réel, elle joue ici un rôle analogue à la perception sensible; elle fournit la matière sensible qui dote les significations générales de déterminations singulières. Perception sensible et imagination « remplissent » de déterminations singulières les intentions de signification généralisantes. Et par là même, elles font advenir de véritables objets là où il n'y avait encore que des concepts.

Certes, comme celles des autres disciples de Brentano, la théorie husserlienne de l'objet est-elle notoirement écartelée entre une conception très large qui admet à titre d' « objets» tout ce qui est représenté, tous les contenus des actes intentionnels - et affirme dès lors qu' « il n'y a pas de 
représentation sans objet »-, et une conception bolzanienne plus étroite qui réserve le terme d'«objet» à ce qui est effectivement donné dans la perception. Dans le premier sens, les significations générales «triangle», «montagne », « licorne » sont déjà des objets du simple fait qu'elles sont pensées, visées par des intentions de signification; dans le second sens, elles ne sont encore que des contenus auxquels des objets dans la réalité peuvent alors correspondre ou non, comme c'est respectivement le cas de «montagne » et « licorne ».

Annonçant Meinong, Twardowski avait fait valoir que, même si elle n'existe pas et n'est pas donnée à la perception sensible, la montagne d'or est déjà bien un objet, car c'est de la montagne d'or elle-même et non du contenu «montagne d'or» que, par exemple, je juge et dis qu'elle est spatialement étendue ${ }^{1}$. Sujet logique de prédications, la montagne d'or est, pour Twardowski, un objet, même si elle n'est qu'un objet immanent auquel ne correspond aucun objet transcendant dans la réalité. Et de même en va-t-il de la licorne. En tant qu'objets immanents, caractérisés par les traits définitoires que leur attribue ma visée - l'un ayant une corne et l'autre pas —, «licorne » et «cheval» ne sont, pour Twardowski, pas très différents l'un de l'autre; la différence tient seulement, dans un second temps, au fait qu'aucun objet transcendant ne correspond à l'objet immanent "licorne » alors qu'il y a des objets transcendants qui correspondent au second. Immanents à la conscience, les objets «licorne » et "cheval» sont comme des tableaux dont l'un est inventé de toutes pièces tandis que l'autre figure la réalité ${ }^{2}$.

Dans son compte-rendu critique de l'ouvrage, Husserl s'était, pour sa part, refusé à dédoubler ainsi objets immanent et transcendant ${ }^{3}$; il n'y pas d'une part l'objet pensé et d'autre part l'objet réel. Beaucoup de commentateurs ont toutefois souligné que Husserl lui-même devrait pourtant réintroduire ultérieurement une distinction similaire sous la forme du doublet $\mathrm{du}$ « noème » et de l'« objet transcendant $»^{4}$. Mais c'est le grand mérite de la

\footnotetext{
${ }^{1}$ K.Twardowski, Zur Lehre vom Inhalt und Gegenstand der Vorstellungen, op. cit., § 6, trad. fr. p. 116.

${ }^{2}$ Ibid., § 4, trad. fr. p. 97-104. Là où Twardowski pense essentiellement la représentation sur le modèle de l'image et de l'imagination, Husserl, dit English (op. cit., p. 173-175), voudra au contraire faire valoir les droits premiers de la signification et les droits derniers de la perception.

${ }^{3}$ E. Husserl, «Intentionale Gegenständen » (1894), en particulier $\S 2$ [Hua XII, p. 303-309], trad. fr. dans Sur les objets intentionnels (1893-1901), op. cit., p. 284-286.

${ }^{4}$ Pour English (op. cit.), Twardowski opposait les corrélats intentionnels de la conscience, qui requièrent l'activité subjective, et les objets « transcendants », qui ne
} 
lecture systématique de l'œuvre husserlienne menée par Jacques English que de montrer que c'est précisément la prise en considération de cette troisième modalité de l'intentionnalité qu'est l'imagination qui a permis à Husserl de repenser le problème de Twardowski en maintenant, contre lui, la distinction du sens et de l'objet (au détriment de celle de l'objet immanent et de l'objet transcendant) tout en réintroduisant la possibilité d'objets seulement imaginés et non réels. Dans sa distinction d'avec la modalité signitive, qui peut viser la signification générale «licorne », la modalité " imaginative », qui peut représenter (avec plus ou moins de précision) une licorne singulière, donne en effet accès non plus à de simples concepts, mais à d'authentiques objets, quoique à des objets inexistants. À cet égard, l'imagination est plus proche de la perception que de l'intention de signification.

Dans le cas de la perception du merle (des Recherches logiques ${ }^{1}$ ) ou de l'arbre (des Idées directrices ${ }^{2}$ ), il n'y a clairement qu'un seul objet, à savoir le merle ou l'arbre réel qui est perçu, même si la conscience a par ailleurs pour contenus, d'une part, le sens (la signification générale) "merle » ou « arbre» qui permet d'appréhender l'objet sous ce concept et, d'autre part, l'ensemble des esquisses par lesquelles cet objet s'est effectivement déjà donné à la conscience, ensemble d'esquisses qui composent l'objet tel qu'il a déjà été perçu, et qui, pour ce qui de lui est immanent à la conscience, est encore indéterminé sur certains aspects qui n'ont pas été perçus, c'est-àdire épistémiquement incomplet. Dans le cas de la simple intention de

la requièrent pas (p. 108), parce qu'il n'avait pas résolu le problème des rapports verticaux entre actes (p. 171-172, 179-180, 206-207) et qu'il s'en tenait à une conception picturale de la représentation (p. 173, 182-183). Cette théorie eut un fort impact sur Husserl, qui la critiqua dès 1894 et dressa contre elle un grand nombre des développements des Recherches logiques (p. 113-115) et en particulier, très explicitement, l'appendice aux $\S \S 11-20$ de la $5^{\mathrm{e}}$ Recherche (p. 124, 184-188). Pour Husserl, il faut reconnaître la transcendance de l'objet même dans le cas de l'imagination. Toutefois, souligne English, en travaillant à la fois sur l'axe des rapports verticaux entre actes et des rapports horizontaux entre les actes et leurs contenus, Husserl allait être amené à accorder un statut ontologique à des intermédiaires entre vécu et réalité, et ce non seulement en ce qui concerne les images, mais aussi et surtout en ce qui concerne les significations (p. 147-148, 157-159). Par ailleurs, Husserl allait bien devoir reconnaître une certaine spécificité de la conscience d'image et quelque chose comme des images internes (p. 205-206).

${ }^{1}$ E. Husserl, Logische Untersuchungen, VI, $\S 4$ [Hua XIX/2, p. 550], trad. fr. op. cit., p. 28-29 ; Cf. aussi l'exemple de l' « encrier» (op. cit., § 6 [Hua XIX/2, p. 558-560], trad. fr. p. 38-40).

${ }^{2}$ E. Husserl, Ideen zu einer reinen Phänomenologie und phänomenologischen Philosophie, $\S \S 88-90$ [Hua III, p. 220-226], trad. fr. op. cit. p. 306-315. 
signification (visée du sens général «merle», « arbre» ou « licorne » sans représentation intuitive qui l'accompagne), il n'y a tout simplement pas d'objet mais seulement un contenu sémantique. Dans le cas de la représentation imaginaire d'une licorne ou d'une montagne d'or singulière, par contre, il y a davantage qu'un contenu sémantique ; il y a aussi un objet, singularisé par sa matière sensible. Et c'est de cet objet de la représentation fictive que l'on dit qu'il est dépourvu d'existence réelle, contrairement au merle ou à l'arbre : «C'est le même centaure Chiron dont je parle à présent et que par là je me représente, qui n'existe pas $»^{1}$. Bien qu'inexistant, le centaure imaginé est, comme l'arbre perçu, un objet plutôt qu'un concept ${ }^{2}$.

Une autre manière de penser la différence entre objets intentionnels et concepts est de dire que la modalité signitive ne donne lieu qu'à des jugements analytiques, tandis que les modalités intuitives (perception et imagination) donnent lieu à des jugements synthétiques. Pour un logicien meinongien comme Parsons, qui caractérise aussi bien objets imaginaires que concepts par l'ensemble des traits constitutifs qui participent de leur définition l'œuvre de Doyle constituant en ce sens une sorte de définition de Sherlock Holmes - , un objet imaginaire ne peut être sujet que de jugements (nucléaires) analytiques: que Sherlock Holmes résolve l'énigme du chien des Baskerville, cela fait partie de ses traits constitutifs, de sa définition, comme il fait partie de la définition d'un triangle qu'il ait trois angles et trois côtés ; et ces deux jugements sont, pour Parsons ${ }^{3}$, analytiques dans le sens le plus strict: ils sont vrais parce que la propriété attribuée au sujet est littéralement incluse en lui comme un des membres de l'ensemble de propriétés qu'il est : Fa ssi $\mathrm{F} \in \mathrm{a}($ avec $\mathrm{a}=\{\mathrm{F}, \mathrm{G}, \mathrm{H}, \ldots\})$.

\footnotetext{
${ }^{1}$ E. Husserl, «Intentionale Gegenständen », § 1 [Hua XII, p. 306], trad. fr. op. cit., p. 283. Reprenant le cas du centaure trente ans plus tard, Husserl amorcera même une réflexion sur la possibilité pour cet objet fictif d'être intersubjectivement partagé ([Hua XXIII, p. 567-568], trad. fr. Phantasia, conscience d'image, souvenir, op. cit., p. 532).

${ }^{2}$ Comme le dit English (op. cit., p. 183, 190-191), Husserl pense la modalité imaginaire à partir de la modalité perceptive (dont elle est une modification) alors que Twardowski faisait l'exact contraire. Que, notamment dans un « dialogue » avec les positions brentaniennes (représentations propres et impropres), Husserl ait songé à rédiger tout un ouvrage sur l'imagination comme modification de la perception, c'est ce qui apparaît clairement dans des manuscrits de 1905, 1916-1917 ou 1921 (cf. à cet égard l'introduction de l'éditeur à Phantasia, conscience d'image, souvenir, op. cit., p. 7, 15, 18-22).

${ }^{3}$ T. Parsons, « Nuclear and extranuclear properties », Noûs, 1978 (vol. 12, n²), 1978, p. 140.
} 
Or, même s'ils décrètent en permanence les propriétés de Holmes sans les tirer d'autre chose que de l'imagination de Doyle, il est clair que les romans de Conan Doyle ne se présentent pas comme une définition de Holmes. Et c'est ce qui montre une fois encore que Holmes n'est pas un concept. Les aventures de Holmes ne se présentent pas comme une suite de jugements analytiques qui stipuleraient les propriétés que Holmes possède par définition, mais comme une série de jugements synthétiques apportant des informations sur certaines propriétés que Holmes est supposé posséder de manière contingente. Tout se passe comme si Doyle ne formulait pas la définition d'un système conceptuel, mais décrivait (partiellement) un monde singulier possible quoique non réel, dont les objets pourraient en principe être perçus, connus par fréquentation (acquaintance), plutôt que seulement conçus.

Du point de vue logique, la logique modale, avec sa sémantique des mondes possibles, semble plus adéquate à traiter la fiction que les logiques meinongiennes ${ }^{1}$. Les mondes possibles de la logique modale sont en effet des analogues du monde réel avec des objets singuliers qui y possèdent des propriétés (y satisfont des fonctions propositionnelles) de manière contingente. À cet égard, les objets du monde réel et des autres mondes se distinguent très nettement de ces «entités » purement théoriques que sont les « objets » caractérisés de dicto, qui n'appartiennent à aucun monde mais sont juste des fonctions trans-monde caractérisées par des traits définitoires et satisfaites par des objets singuliers différents d'un monde à l'autre. Prise de dicto, la femme de Bruno Leclercq est simplement, par définition, la personne quelle qu'elle soit qui a épousé Bruno Leclercq, fonction que des individus singuliers distincts peuvent réaliser dans divers mondes possibles. Que la femme de Bruno Leclercq, prise en ce sens générique, ait épousé Bruno Leclercq est analytique et nécessairement vrai, vrai dans tous les mondes possibles; et, à quelques autres conséquences analytiques près, c'est tout ce qui est vrai d'elle. Prise de re, par contre, la femme de Bruno Leclercq, c'est Catherine Fauville et elle a un tas de propriétés qui se distinguent de celle d'avoir épousé Bruno Leclercq et qui ne découlent pas d'elle ; un tas de jugements synthétiques sont vrais à son égard. Bien plus, on peut tout à fait dire d'elle que - si elle avait eu un peu plus de clairvoyance - elle aurait pu ne pas épouser Bruno Leclercq ; contrairement à ce qui est le cas de cette " entité » théorique qu'est l'épouse de Bruno Leclercq prise de dicto, il est contingent de l'épouse de Bruno Leclercq prise de re qu'elle ait

${ }^{1}$ B. Leclercq, « En matière d'ontologie, l'important n'est pas de gagner, mais de participer », art. cit., p. 1-24. 
épousé Bruno Leclercq. Prise de altro mundo, c'est-à-dire en tant qu'objet imaginaire, l'épouse de Bruno Leclercq, c'est celle qui, par exemple, habite ses rêves et ses fantasmes; et, si elle se distingue de l'épouse réelle par certaines propriétés, elle est, comme elle, un objet singulier, qui possède, comme elle, des propriétés contingentes, c'est-à-dire qui ne découlent pas trivialement de la simple définition d'être l'épouse de Bruno Leclercq. Là où l'épouse de Bruno Leclercq prise de dicto n'est qu'un concept qui se réduit à des traits définitoires et à leurs conséquences analytiques, de sorte qu'elle n'est l'objet d'aucun désir que ne satisfait déjà l'épouse réelle ou que ne satisferait de la même manière n'importe quelle épouse de Bruno Leclercq ${ }^{1}$, l'épouse imaginaire, par contre, est un objet singulier de désir parce qu'elle est riche d'une certaine matérialité sensible (imaginée et non perçue) qui la rend particulièrement intéressante.

À l'encontre de toute une littérature sur l'intentionnalité, qui, sous la catégorie générale d'objet intentionnel ou d'objet immanent, et au prétexte de l'inexistence réelle des uns et des autres, n'a cessé de brouiller la distinction entre concepts, qui sont les corrélats - généraux — de simples intentions de signification, et objets imaginaires, qui sont les corrélats — singuliers — de ces actes intuitifs que sont les représentations imaginaires, il convient donc, comme l'a justement souligné Jacques English, de redonner toute sa valeur à l'écart entre deux types d'actes intentionnels très différents, et corrélativement entre deux types de contenus intentionnels très différents, les contenus sémantiques et les objets imaginaires.

\section{Le concevable et l'imaginable}

Aussitôt ce pas effectué avec Husserl (lu par English), il faut toutefois compliquer immédiatement ce que nous avons dit jusqu'à présent de la distinction entre jugements analytiques et synthétiques, et donc aussi de ce que nous

\footnotetext{
${ }^{1}$ Je peux bien sûr entretenir certains désirs à l'égard de l'épouse de Bruno Leclercq en général (prise de dicto) au sens où je peux simplement désirer être marié et avoir une épouse quelle qu'elle soit, ou désirer que mon épouse quelle qu'elle soit aime les voyages ou les enfants. Toutefois, si on y regarde bien, ce ne sont pas là des désirs à l'égard du concept, mais plutôt à l'égard d'objets singuliers qui, dans tel ou tel monde, satisferaient ce concept : cela n'a pas de sens de vouloir de l'épouse de Bruno Leclercq en général qu'elle aime les enfants ; l'épouse de Bruno Leclercq en général ne peut avoir d'autres propriétés que celles qui découlent de sa définition; seule une épouse singulière peut posséder cette propriété intéressante d'aimer les enfants.
} 
avons dit de la distinction entre concepts et objets, pour prendre toute la mesure des rôles épistémologiques de l'intention de signification et de l'imagination tels que s'efforce de les penser le père de la phénoménologie.

Que le triangle en général ait trois côtés relève de sa seule définition; c'est là un jugement purement analytique. Mais qu'en est-il de ces jugements, pointés par Kant comme "synthétiques a priori», selon lesquels «Aucun des trois côtés d'un triangle ne peut être plus long que la somme de la longueur des deux autres» ou «La somme des angles d'un triangle équivaut à $180^{\circ} »$ ?

Pour Kant ${ }^{1}$, l'analyse du concept de triangle ne suffit pas à nous fournir cette propriété qu'aucun des trois côtés ne puisse être plus long que la somme de la longueur des deux autres, alors que c'est par contre une chose absolument évidente dès que l'on cherche à fournir une représentation sensible d'un triangle. Ici, les contraintes mêmes de la représentation intuitive du triangle dans l'espace semblent fournir une information qui ne découlait pas des seules contraintes de la définition du concept.

La chose est encore plus claire avec l'information relative à la somme des angles. Que la somme des angles d'un triangle soit équivalente à $180^{\circ}$, ce n'est, savons-nous depuis Bolyai, Lobatchevski et Riemann, pas du tout une nécessité analytique résultant de la définition du triangle; les géométries hyperboliques (ou plus généralement à courbure négative) prévoient des triangles dont la somme des angles est inférieure à $180^{\circ}$, tandis que les géométries elliptiques (ou plus généralement à courbure positive) prévoient des triangles dont la somme des angles est supérieure à $180^{\circ}$. Kant avait-il pour autant raison de voir dans l'énoncé de la somme des angles d'un triangle un jugement synthétique a priori ? Kant n'avait certes pas anticipé que l'on puisse un jour concevoir des triangles non-euclidiens. Mais il avait souligné que concevoir intellectuellement, par de simples intentions de signification, est autre chose que représenter intuitivement dans l'espace sensible. Or, s'il s'avérait que, contrairement à la pure conception, qui peut envisager des plans de courbures diverses, la représentation spatiale intuitive (perceptuelle ou imaginaire) était pour sa part soumise aux contraintes de plans à courbure nulle, il serait alors nécessairement vrai que les triangles intuitivement représentés ont une somme d'angle égale à $180^{\circ}$, et ce même si les triangles conçus n'ont pas forcément tous cette propriété. On peut bien sûr douter que toute représentation spatiale intuitive soit soumise aux contraintes de plans à courbure nulle - certes, les feuilles de papier et les tableaux sur

${ }^{1}$ I. Kant, Kritik der reinen Vernunft, A25/B39, trad. fr. Critique de la raison pure, Paris, Gallimard, 1980, p. 92. 
lesquels les mathématiciens dessinent leurs figures sont de facto de tels plans, mais est-ce forcément le cas de toute représentation spatiale intuitive possible, même imaginaire ? - , mais ce que Kant met là en évidence, et que Husserl s'efforcera de penser au moyen de la distinction entre l'a priori formel (ou analytique) et l'a priori matériel (ou synthétique), c'est la possibilité même de distinguer entre des contraintes relevant de la pure pensée conceptuelle et des contraintes qui s'ajouteraient aux premières et qui relèveraient pour leur part des conditions de possibilité de la représentation intuitive.

Comme nous l'avons montré ailleurs ${ }^{1}$, ce qui se joue là, dans la distinction entre les limites du concevable et les limites de l'imaginable, ce sont évidemment une fois encore les rapports entre deux types d'actes de représentation - les intentions de signification, qui caractérisent la simple conception, et les actes de leur remplissement intuitif, qu'il soit perceptif ou imaginaire - et leurs apports respectifs à la théorie de la connaissance, mais aussi, à travers ces rapports, le statut même de la phénoménologie dans sa distinction notamment de la philosophie analytique. L'analyse conceptuelle qui préside à cette dernière permet d'explorer la sphère de l'analytique, c'està-dire des nécessités et possibilités régissant le pensable (ou concevable), mais la phénoménologie se donne aussi pour objectif d'explorer les nécessités et possibilités régissant l'intuitivement représentable. Or, à cet égard, l'imagination joue un rôle essentiel, car ce qui est représentable, ce n'est pas seulement ce qui est effectivement perçu, mais ce qui peut être perçu, ce qui pourrait faire l'objet d'une expérience sensible. Au-delà de l'effectif, l'imagination est un formidable outil d'investigation des possibles $\mathrm{du}$ représentable, lesquels ne se réduisent donc pas aux possibles du concevable (du pensable), possibles logiques que l'intention de signification, soumise au principe de non-contradiction, suffit à étudier ${ }^{2}$.

Dans les expériences de pensée que font les philosophes pour investiguer le possible et, par là même, l'impossible et le nécessaire, il y a en fait deux sortes nettement distinctes : il y a celles qui se contentent d'envisager ce qui est logiquement concevable et ne viole pas les lois formelles de la pensée, donc ce qui est consistant, non-contradictoire; et il y a celles qui

\footnotetext{
${ }^{1}$ B. Leclercq, «Le concevable et l'imaginable. Analyse conceptuelle et intuition éidétique », dans Philippe Cabestan (éd.), Claude Romano. La raison et l'événement, le Cercle herméneutique, 2016, p. 111-132.

${ }^{2}$ Sur ce point, voir plus particulièrement le texte «Pure possibilité et phantasia » de Phantasia, conscience d'image, souvenir, op. cit., p. 525-526 [Hua XXIII, p. 559$561]$.
} 
envisagent en outre, au sein de ce qui est concevable, la distinction entre ce qui est intuitivement représentable, imaginable, et ce qui ne l'est pas. Les variations imaginaires que Husserl met au fondement de l'intuition eidétique relèvent de la seconde catégorie et c'est pourquoi elles ont une portée phénoménologique et non simplement logique, analytique.

À cet égard, les travaux sémio-logiques de Charles Sanders Peirce ont bien montré le rôle que jouent les explorations de l'imagination dans la constitution des intuitions «pures » de la forme spatiale sur lesquelles Kant fonde les jugements synthétiques a priori de la géométrie ${ }^{1}$. Une démonstration comme celle de la proposition que la somme des angles d'un triangle équivaut à $180^{\circ}$ requiert, disait $\mathrm{Kant}^{2}$, des «constructions », dont Peirce montre qu'elles impliquent la mise à l'épreuve imaginaire des contraintes mêmes de la représentation spatiale ${ }^{3}$. Seule l'expérience de ces contraintes de la représentativité spatiale permet d'obtenir des informations sur le triangle qui ne sont pas seulement analytiques mais nouvelles et théorématiques. En tant que science de l'espace représentable, la géométrie est une discipline irrémédiablement fondée sur l'intuition sensible, non pas tant perceptive (ce qui en ferait une discipline empirico-inductive) qu'imaginative (ce qui lui permet d'explorer les possibles et par là même de dégager des nécessités a priori).

Dans cette perspective, il convient dès lors sans doute de distinguer deux types de géométrie : l'une qui définit théoriquement, c'est-à-dire de manière purement conceptuelle, des objets théoriques que, pour reprendre les mots de Hilbert, "on peut bien, si on veut, nommer "points", "droites",

\footnotetext{
${ }^{1}$ Voir sur ce point B. Leclercq, «Are there synthetic a priori propositions? The paradigmatic case of mathematics, from Kant to Frege and Peirce », à paraître dans Vesselin Petrov (éd.), Mathematics in Philosophy, Louvain-la-Neuve, Chromatika, 2016; « Iconic virtues of diagrams. Peirce on ampliative reasoning », à paraître dans Signata dans un volume consacré aux textes du Congrès Theorein. The Influence of Pictures on Knowledge and Vice Versa, 2017.

${ }^{2}$ I. Kant, Kritik der reinen Vernunft, A716-717/B744-745, trad. fr. op. cit., p. 606.

${ }^{3}$ C. S. Peirce, Collected Papers of Charles Sanders Peirce, vols. 1-6, Charles Hartshorne and Paul Weiss (éds.), 1931-1935, vols. 7-8, Arthur W. Burks (éd.), 1958, Cambridge, Harvard University Press, vol. 1, p. 54, 238-240 ; vol. 2, p. 216, 443-444, 601, 778, 782 ; vol. 3, p. 207, 424, 560, 641 ; vol. 5, p. 490. Writings of Charles S. Peirce, A Chronological Edition, Peirce Edition Project (éds.), Indiana University Press, Bloomington and Indianapolis, 1982 suiv., vol. 5, p. 164, 332 ; vol. 6, p. 37, 258-259, 355 ; vol. 8, p. 17, 24.
} 
"plans" " ${ }^{1}$, et qui en tire toutes les conséquences analytiques (produisant ainsi, selon la définition initiale des concepts, des géométries euclidiennes ou non-euclidiennes, archimédiennes ou non-archimédiennes, ...) ; l'autre qui se préoccupe des conditions de la représentation spatiale intuitive et qui ne repose donc pas seulement sur l'analyse logico-conceptuelle avec ses principes de non-contradiction et de conséquence déductive, mais s'appuie également sur des «intuitions sensibles» effectives et imaginaires, dont la valeur générique tient précisément dans le fait qu'elle n'inclut pas seulement des expériences sensibles effectives mais envisage également, par variations imaginaires, les expériences sensibles possibles.

Or, c'est exactement ce double niveau de contraintes - conceptuelles et intuitives - qu'après avoir d'abord univoquement rangé la géométrie parmi les disciplines régionales (science des objets spatiaux) relevant de l'a priori matériel (par opposition à l'a priori analytico-formel), Husserl ${ }^{2}$ viendra à théoriser sous la forme de la distinction entre logique de la consistance (et de la conséquence analytique) et logique de la vérité possible, c'est-à-dire de l'intuitivité possible; la première est entièrement analytique, tandis que la seconde explore les conditions de la représentation intuitive et est proprement phénoménologique. Il se pourrait ainsi que les géométries non euclidiennes soient consistantes (non-contradictoires) mais pas conformes à l'intuition; qu'un triangle non euclidien, dont la somme des angles n'équivaudrait pas à $180^{\circ}$, soit parfaitement concevable (et puisse faire l'objet d'investigations conceptuelles, c'est-à-dire déductives), mais pas intuitivement représentable (pas imaginable); ou, pour le dire encore autrement, il se pourrait que cette propriété de la somme des angles d'un triangle d'être équivalente à $180^{\circ}$ ne soit pas une nécessité conceptuelle, c'est-à-dire pas prescrite par la définition du triangle, mais qu'elle soit néanmoins une nécessité intuitive, c'est-à-dire prescrite par les conditions de la représentation sensible, conditions que met en évidence l'imagination en tant qu'elle ne se limite pas à la représentation sensible effective, la perception, mais inclut aussi la représentation sensible possible...

\footnotetext{
${ }^{1}$ D. Hilbert, Grundlagen der Geometrie, trad. fr. Fondements de la géométrie, Paris, Dunod, 1971, p. 11.

${ }^{2}$ E. Husserl, Formale und Transzendentale Logik, §§ 15 sq. [Hua XVII, p. 60 sq.], trad. fr. Logique formelle et logique transcendantale, Paris, P.U.F., 1957, p. 79 sq.
} 


\section{Concepts, objets singuliers et objets généraux}

Tout ceci nous ramène à la distinction des concepts et des objets pour tout à la fois la confirmer et la nuancer.

Les remarques que nous avons formulées jusqu'ici confirment la nécessité de distinguer nettement différents types de contenus intentionnels, ainsi que différents modes de leur inexistence mais aussi de leur impossibilité. Un triangle à quatre côtés est conceptuellement impossible ; c'est une contradiction analytique. Mais un triangle dont la somme des angles est supérieure à $180^{\circ}$ est concevable, au sens où il peut être défini (et visé par des intentions de signification) sans contradiction logique ; il n'est par contre pas intuitivement représentable si toute représentation sensible suppose des plans à courbure nulle. Un triangle dont la somme est supérieure à $180^{\circ}$ est alors intuitivement impossible, inimaginable, quoique pas conceptuellement impossible, pas inconcevable ${ }^{1}$.

En pensant les objets incomplets et les objets impossibles - et donc les violations des principes logiques du tiers exclu et de la non-contradiction — en opposition au « préjugé en faveur de l'actuel » (Meinong), les logiques meinongiennes ont prétendu rendre compte de la légalité propre à une sphère d'objets (intentionnels) plus large que celle des objets réels. Mais c'est ce qui s'est fait au prix de lourdes confusions : il y a en fait plusieurs incomplétudes et plusieurs impossibilités. L'incomplétude épistémique de l'objet réel partiellement perçu ou de l'objet imaginaire partiellement représenté n'est pas du même ordre que l'incomplétude principielle du concept ; et l'impossibilité analytique (sous peine de contradiction) qui caractérise les conditions de la pensée (conception par intentions de signification) n'est pas du même ordre que l'impossibilité synthétique a priori qui régit les conditions de la représentation intuitive. Le triangle à quatre côtés est principiellement incomplet et analytiquement impossible; le triangle dont la somme des angles est supérieure à $180^{\circ}$ est principiellement incomplet et matériellement impos-

\footnotetext{
${ }^{1}$ Pour sa part, Husserl (Logische Untersuchungen, IV, § 12 [Hua XIX/1, p. 334], trad. fr. Recherches Logiques, Paris, P.U.F., 1961, vol. 2, p. 121) prend pour exemple d'impossibilité matérielle (synthétique) le carré rond, qu'il estime concevable quoique non représentable, et qui se distinguerait ainsi du carré non rectangulaire, lequel est logiquement contradictoire, donc formellement, analytiquement, impossible et tout simplement inconcevable. Que l'on ne puisse tirer, des définitions du cercle et du carré, aucune incompatibilité analytique ne paraît toutefois pas évident. Nous utiliserons donc ici l'exemple, moins contestable, du triangle dont la somme des angles est supérieure à $180^{\circ}$, lequel n'est clairement pas analytiquement impossible.
} 
sible ; le triangle en général est principiellement incomplet et analytiquement autant que matériellement possible; de même en va-t-il de la licorne en général ; telle licorne singulière, par contre, est analytiquement et matériellement possible et principiellement complète quoique épistémiquement incomplète en tant qu'objet partiellement imaginé (et décrit) ; et il en va de même de Sherlock Holmes ; quant à l'objet réel Bruno Leclercq, il est analytiquement et matériellement possible et principiellement complet quoique épistémiquement incomplet en tant qu'objet perçu par esquisses. Il reste évidemment très intéressant de développer des logiques paraconsistantes (violant le principe de non-contradiction) et/ou paracomplètes (violant le principe du tiers-exclu) pour traiter de ces différents types d' «objets » et de la manière dont ils peuvent se voir attribuer des propriétés dans des jugements. Mais le cadre des logiques meinongiennes, qui écrase les distinctions entre concepts et objets fictifs, entre l'incomplétude principielle des premiers et l'incomplétude épistémique (représentation intuitive incomplète) des seconds, mais aussi entre impossibilité conceptuelle et impossibilité intuitive - ce que Husserl appelle contresens formel (ou contradiction logique) et contresens matériel — ne semble pas adéquat.

Ce que nous avons dit de l'a priori matériel et des variations imaginaires qui permettent de le mettre en évidence impose toutefois également de nuancer l'opposition nette faite entre concepts et objets. En tant qu'il est défini comme figure fermée à trois côtés droits dont les propriétés se limitent aux conséquences analytiques de cette définition, le triangle en général n'est que le corrélat d'une intention de signification, donc un simple concept, contrairement aux triangles singuliers que je me représente intuitivement dans la perception ou l'imagination (triangles qui ont une taille, une forme, une couleur, qui ne sont pas prescrites par la définition). Mais, entre le concept de triangle et les triangles singuliers représentés dans l'intuition, il semble qu'il y ait place pour le triangle représentable en général, celui dont la démonstration kantienne montre que la somme de ses angles équivaut à $180^{\circ}$ (et qui ne s'identifie donc pas au concept de triangle, lequel n'a pas cette propriété). Ce triangle représentable en général fait, pour Kant, l'objet d'une intuition pure, et Peirce montre ce que, dans sa prétention à valeur universelle et même nécessaire, une telle intuition doit aux explorations de l'imagination. De la même façon Husserl parle d' «intuition eidétique » reposant sur des variations imaginaires ${ }^{1}$. Mais, s'il fait ainsi l'objet d'une expérience propre, d'une «intuition » qui le dote de propriétés qui ne dé-

1 E. Husserl, Ideen zu einer reinen Phänomenologie und phänomenologischen Philosophie, $\S \S 4,70$ [Hua III, p. 16-17, 160-163], trad. fr. op. cit. p. 24-25, 223-227. 
coulent pas analytiquement de sa définition, le triangle représentable, quoique général, n'est plus un simple concept; c'est un objet, objet général que Husserl appelle une "essence ${ }^{1}$ et qui est donc nettement distinct d'un concept.

\section{Bibliographie}

Bolzano Bernard, Wissenschaftslehre, trad. fr. partielle Théorie de la science, Paris, Gallimard, 2011.

Derrida Jacques, La voix et le phénomène, Paris, P.U.F., 1967.

English Jacques, Sur l'intentionnalité et ses modes, Paris, P.U.F, 2006.

Hilbert David, Grundlagen der Geometrie, trad. fr. Fondements de la géométrie, Paris, Dunod, 1971.

Husserl Edmund, «Intentionale Gegenständen », 1894 in Aufsätze und Rezensionen (1890-1910) [Hua XII, p. 303-348], trad. fr. « Objets intentionnels (1894) », dans E. Husserl - K. Twardowski, Sur les objets intentionnels (1893-1901), Paris, Vrin, 1993, p. 279-326.

Husserl Edmund, Formale und Transzendentale Logik, 1929 [Hua XVII], trad. fr. Logique formelle et logique transcendantale, Paris, P.U.F., 1957.

Husserl Edmund, Ideen zu einer reinen Phänomenologie und phänomenologischen Philosophie, 1913 [Hua III], trad. fr. Idées directrices pour une phénoménologie I, Paris, Gallimard, 1950.

Husserl Edmund, Logische Untersuchungen, 1900-1901 [Hua XIX], trad. fr. Recherches Logiques, Paris, P.U.F., 1959-1963

Husserl Edmund, Phantasie, Bildbewußtsein, Erinnerung. Zur Phänomenologie der anschaulichen Vergegenwärtigungen. Texte aus dem Nachlaß (1898-1925) [Hua XXIII], trad. fr. Phantasia, conscience d'image, souvenir, Grenoble, Millon, 2002.

Kant Immanuel, Kritik der reinen Vernunft, 1781/1787, trad. fr. Critique de la raison pure, Paris, Gallimard, 1980.

Leclercq Bruno, «À l'impossible, nul objet n'est tenu. Statut des 'objets' inexistants et inconsistants et critique frégéo-russellienne des logiques meinongiennes", dans Sébastien Richard éd., Analyse et ontologie. Le renouveau de la métaphysique dans la tradition analytique, Paris, Vrin, 2011, p. 159-198.

Leclercq Bruno, «Are there synthetic a priori propositions? The paradigmatic case of mathematics, from Kant to Frege and Peirce », à paraître dans Vesselin Petrov (éd.), Mathematics in Philosophy, Louvain-la-Neuve, Chromatika, 2016.

\footnotetext{
${ }^{1}$ Sur les rapports des possibilités d'individuel (ex. objets de fiction) aux essences, entendues comme généralités saisissables individuellement, cf. [Hua XXIII, p. 569570], trad. fr. Phantasia, conscience d'image, souvenir, op. cit., p. 534.
} 
Leclercq Bruno, «En matière d'ontologie, l'important n'est pas de gagner, mais de participer », dans Igitur, 2012 (vol. 4, n²), p. 1-24.

Leclercq Bruno, «Faire cohabiter les objets sans domicile fixe (homeless objects). Chisholm et les logiques meinongiennes », dans Bulletin d'Analyse Phénoménologique, 2014 (vol. 10, $\mathrm{n}^{\circ}$ 6), D'un point de vue intentionnel : Aspects et enjeux de la philosophie de Roderick Chisholm (Actes n ${ }^{\circ}$ 6), p. 85-111.

Leclercq Bruno, "Iconic virtues of diagrams. Peirce on ampliative reasoning ", à paraître dans Signata, volume consacré aux textes du Congrès Theorein. The influence of pictures on knowledge and vice versa, 2017.

Leclercq Bruno, «Le concevable et l'imaginable. Analyse conceptuelle et intuition éidétique ", dans Philippe Cabestan (éd.), Claude Romano. La raison et l'événement, le Cercle herméneutique, 2016, p. 111-132.

Leclercq Bruno, «Quand c'est l'intension qui compte. Opacité référentielle et objectivité », dans Bulletin d'Analyse Phénoménologique, 2010 (vol. 6, $\mathrm{n}^{\circ}$ 8), p. 83-108.

Meinong Alexius, Über Gegenstandstheorie, Leipzig, Barth, 1904, trad. fr. La théorie de l'objet, Paris, Vrin, 1999.

Meinong Alexius, Über Möglichkeit und Warscheinlichkeit, Leipzig, Barth, 1915.

Parsons Terence, « Nuclear and extranuclear properties », Noûs, 1978 (vol. 12, n²), 1978, p. 137-151.

Parsons Terence, Nonexistent objects, New Haven \& London, Yale University Press, 1980.

Peirce Charles Sanders, Collected Papers of Charles Sanders Peirce, vol. 1-6, Charles Hartshorne and Paul Weiss (éds.), 1931-1935 ; vol. 7-8, Arthur W. Burks (éd.), 1958, Cambridge, Harvard University Press

Peirce Charles Sanders, Writings of Charles S. Peirce, A Chronological Edition, Peirce Edition Project (éds.), Indiana University Press, Bloomington and Indianapolis, 1982.

Routley Richard, Exploring Meinong's Jungle and Beyond, Canberra, Department Monograph \#3 of the Philosophy Department of the Australian National University, 1980.

Twardowski Kazimierz, Zur Lehre vom Inhalt und Gegenstand der Vorstellungen, 1894, trad. fr. Sur la théorie du contenu et de l'objet des représentations, dans E. Husserl - K. Twardowski, Sur les objets intentionnels (1893-1901), Paris, Vrin, 1993. 